\title{
Population-based family case-control proband study on familial aggregation of metabolic syndrome: Finding from Taiwanese people involved in Keelung community-based integrated screening (KCIS no. 5)
}

\author{
Yueh-Hsia Chiu $^{\mathrm{a}, \mathrm{b}}$, Wen-Yuan Lin ${ }^{\mathrm{a}}$, Po-En Wang ${ }^{\mathrm{b}}$, Yao-Der Chen ${ }^{\mathrm{c}}$, \\ Ting-Ting Wang ${ }^{\mathrm{b}}$, Jane Warwick ${ }^{\mathrm{d}}$, Tony Hsiu-Hsi Chen ${ }^{\mathrm{a}, \mathrm{e}, *}$ \\ a Institute of Preventive Medicine, College of Public Health, National Taiwan University, Taipei, Taiwan \\ ${ }^{\mathrm{b}}$ Health Bureau of Keelung City, Keelung City, Taiwan \\ ${ }^{\mathrm{c}}$ Health Bureau of Tainan County, Tainan, Taiwan \\ ${ }^{\mathrm{d}}$ Cancer Research UK Centre for Epidemiology, Mathematics and Statistics, Wolfson Institute of Preventive Medicine, \\ Charterhouse Square, London, UK \\ ${ }^{\mathrm{e}}$ Division of Biostatistics, Graduate Institute of Epidemiology, College of Public Health, \\ National Taiwan University, Taipei, Taiwan
}

Received 22 May 2006; accepted 12 July 2006

Available online 28 August 2006

\begin{abstract}
A population-based case-control proband study was undertaken to elucidate familial aggregation, independent environmental factors, and the interaction between them. A total of 7308 metabolic syndrome (MET-S) cases were identified from the Keelung community-based integrated screening programme between 1999 and 2002. The study has a case-control/family sampling design. A total of 1417 case probands were randomly selected from 3225 metabolic syndrome cases and the corresponding 2458 controls selected from 16,519 subjects without metabolic syndrome by matching on sex, age ( \pm 3 years) and place of residence. The generalized estimation equation model was used to estimate odds ratios and corresponding $95 \%$ confidence intervals. The risk for having metabolic syndrome among family members for cases versus control probands was 1.56-fold (1.29-1.89) after controlling for significant environmental factors. Higher risk of metabolic syndrome was found in parents than spouse. Low education against high education had 2.06-fold (1.36-3.13) risk for metabolic syndrome. Betel quid chewing was positively associated with the risk of MET-S, with 1.99-fold (1.13-3.53) risk for 1-9 pieces and 1.76-fold (0.96-3.23) risk for $\geq 10$ pieces compared with non-chewer. Moderate and high intensity of non-occupational exercise led to $21.0 \%(\mathrm{OR}=0.79(0.63-0.98))$ and $26.0 \%(\mathrm{OR}=0.74(0.59-$ 0.94)) reduction in the risk for metabolic syndrome, respectively. The frequent consumption of vegetable reduced $24.0 \%$ $(\mathrm{OR}=0.76(0.62-0.92))$ risk for MET-S. The frequent consumption of coffee was associated the increased risk for metabolic syndrome $(\mathrm{OR}=1.32(1.07-1.64))$. The present study confirmed the risk of metabolic syndrome not only has the tendency towards familial aggregation but is affected by independent effect of environmental or individual correlates.
\end{abstract}

(C) 2006 Elsevier Ireland Ltd. All rights reserved.

Keywords: Metabolic syndrome; Family case-control proband study; Familial aggregation; Environmental factors

\footnotetext{
* Corresponding author at: Division of Biostatistics, Graduate Institute of Epidemiology/Institute of Preventive Medicine, College of Public Health, National Taiwan University, Room 521, No. 17, Hsuchow Road, Taipei 100, Taiwan. Tel.: +886 233228021 ; fax: +886 223587707 .

E-mail address: stony@episerv.cph.ntu.edu.tw (T.-H. Chen).
} 


\section{Introduction}

Metabolic syndrome (MET-S) is characterized by a variety of metabolic conditions including hyperglycemia, high blood pressure, hyperlipidemia, and central obesity. The clustering of these components indicates risk factors associated with MET-S have common underlying genetic and/or environment factors. Previous studies show that the occurrence of MET-S is positively associated with low educational attainment [1-5], low levels of physical activity [6-8], and "western diet" [9], and inversely associated with dairy consumption [10], which are all specific to the individual. Several studies have shown the clustering of mean blood pressure and high density lipoprotein cholesterol to be stable from childhood to adulthood [11,12]. Prior studies have also shown relative contributions from genetic factors and environmental factors to the development of MET-S using classical twin studies [13-15] or family studies based on modeling technique [16] or a genome-wide linkage scan method [17]. While these western studies have been conducted to assess genetic and environmental factors related to the development of MET-S $[18,19]$ there is paucity of population-based family studies on the elucidation of genetic and environment factors, particularly seen in Chinese people.

As we are tempted to assess family aggregation of MET-S by taking other individual correlates or environmental factors into account, we therefore decided upon a case-control/family sampling design using data derived from a large population-based cohort to elucidate familial aggregation and potential shared or non-shared environmental factors simultaneously.

\section{Materials and methods}

\subsection{Study population}

Study subjects were identified from the Keelung (the northernmost city in Taiwan) community-based integrated screening programme (KCIS) during the period 1999-2002. Details of the study design and preliminary results from KCIS during its first 3 years (1999-2001) are described elsewhere [20]. Following the success of the first 3-year project, the KCIS programme was extended to cover 58,669 residents ( $20.3 \%$ of the population aged over 20 years) up until the end of 2002. Of these, 43,158 have complete information on high density lipoprotein (HDL) and low density lipoprotein (LDL) and it is these subjects that form the basis of the following analysis. The 43,158 subjects relate to 32,522 households and include 7308 subjects with metabolic syndrome as determined by the modified NCEP ATPIII criteria [21]. Abdominal obesity was redefined as waist circumference $\geq 90 \mathrm{~cm}$ in men and $\geq 80 \mathrm{~cm}$ in women, following recommendations from the Asia-Pacific conference [22,23].

\subsection{Study design}

Our study design is based on the case-control/family sampling design proposed by Liang and Pulver [24]. This design is intended to assess familial aggregation by identifying both case and control probands and comparing the distribution of MET-S amongst their family members. Fig. 1 shows a flow chart of the overall study design that gives rise to 1716 family members of case probands (278 subjects with MET-S and 1438 subjects without MET-S) and 2974 family members of the control probands (294 subjects with MET-S and 2680 subjects without MET-S).

\subsection{Data collection}

Biochemical and anthropometric variables for defining MET-S were collected through on-site screening for each participant in the KCIS programme. A venous blood sample for measuring plasma glucose was taken after 12-h fasting, including triglycerides, total cholesterol, LDL, HDL, complete blood count, alanine transaminase, aspartate transaminase, albumin, total protein, blood urea, nitrogen, creatinine, uric acid, and so on.

Regarding anthropometric factors, trained staff measured height, waist, and hip circumference (measured to nearest $0.1 \mathrm{~cm}$ ) and weight (measured to the nearest $0.1 \mathrm{~kg}$ ) to obtain body mass index (BMI), the waist/hip circumference ratio (WHR) was also calculated. The same staff measured blood pressure in the right arm using an appropriately sized cuff and a standard mercury sphygmomanometer.

Information on life style factors for participants in the KCIS programme were collected through face-to-face interview using a structured questionnaire administered by public health nurses. Besides demographic characteristics, the questionnaire included five major parts; lifestyle habits (smoking, alcohol intake, betel quid chewing, and level of physical activity), daily diet (intake of meat, soybean, vegetable, fruit, milk, and coffee drink), family history of cancer and chronic diseases, personal medical history, and female reproductive experiences (age at menarche, age at menopause, and parity). Smoking status was classified as never, current, and quit smoking. The habits of drinking alcohol and betel nut chewing were defined in the same manner. Also collected were the exposure factors with respect to age at commencement, duration, and quantity of cigarettes, alcohol, and betel quids consumed. Physical activity was defined as nonoccupational exercise with information on the number of times per week and duration (in minutes). In the following analysis, the frequency and intensity of physical activity were categorized by tertitle distribution (see below). We calculated physical activity with by (times of per week) $\times$ (minutes of per time).

Diet during the past 6 months (including sea food, meat, fish, fried oil, bean or egg products, fruit and vegetables, milk, soda drinks, and coffee) was also obtained. We displayed the food moulds and standard dishes or containers of each food to 
illustrate the proportion each food was consumed at one time. Frequency of consumption was then categorized into five groups: never or seldom, 1-2 times per week, 3-4 times per week, 5-6 times per week, and more than 7 times per week. For the analysis, we defined high level of intake as being more than five times per week.

\subsection{Statistical methods}

Since our study has a case-control/family sampling design the presence of MET-S among family members of the same proband may be correlated. Therefore, the assumption of independence, implicit in the conventional statistical methods, may be inappropriate. We therefore used a statistical model based on the Liang and Pulver method [24] that enables one to assess familial aggregation, environmental risk factors and the relevant interactions allowing for this correlation. Note that although our study is a matched case-control/family sampling design, both matched and unmatched analyses with the incorporation of matching factors have been performed. As the results were similar, whether or not the matching was respected in the analysis, we present only the unmatched

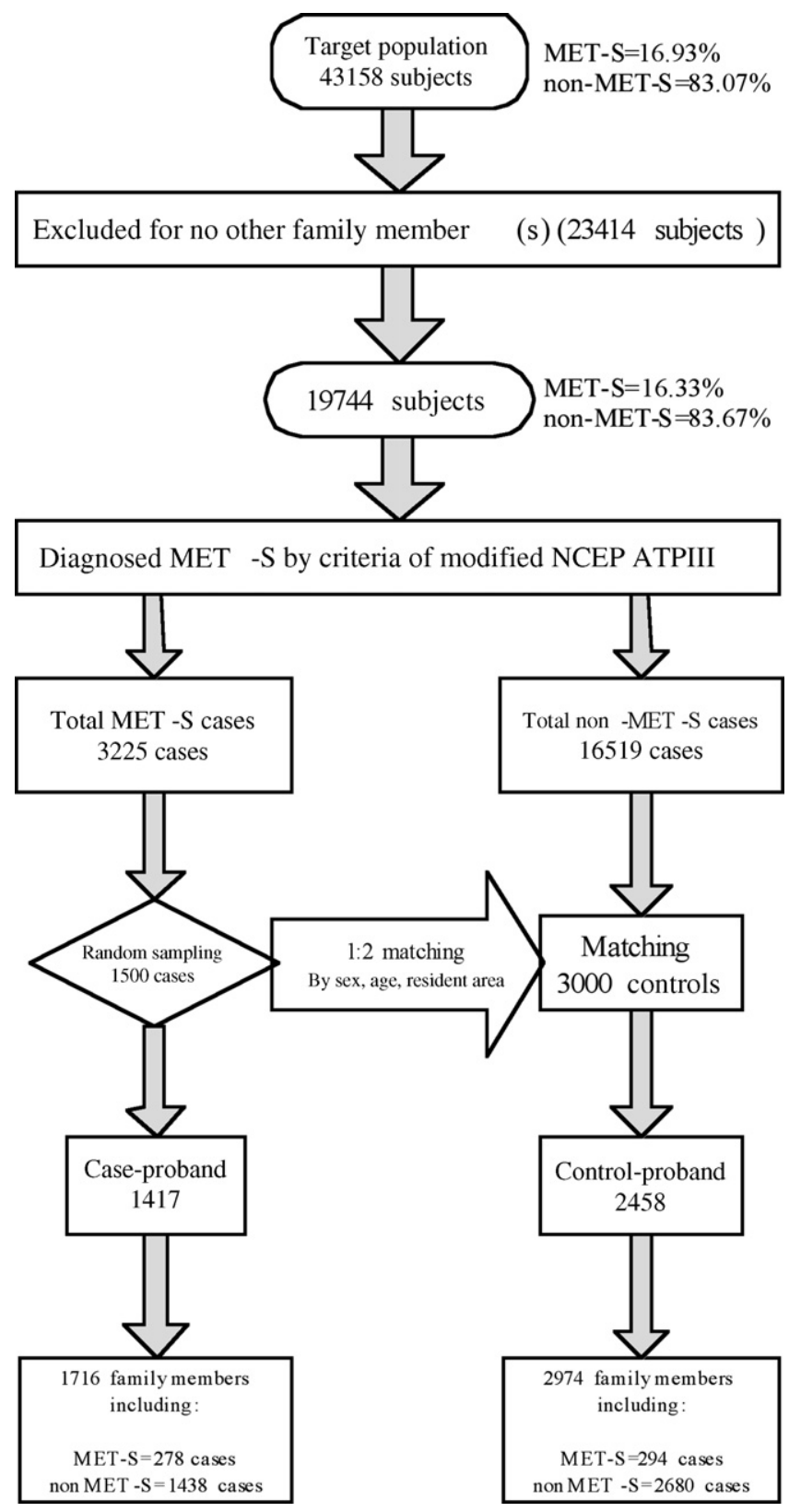

Fig. 1. Study design of case-control proband study on metabolic syndrome. 
logistic regression model. A detailed description of this mathematical model is given in Appendix A. In this model, the response variable $(y)$ is the presence $(y=1)$ or absence $(y=0)$ of MET-S among relatives of the cases or controls, and the independent variable status $(z)$ which is set to 1 for case probands and their family members and to 0 for control probands and their family members, indicating familial aggregation. Environmental and other risk factors $\left(x_{1}-x_{\mathrm{k}}\right)$ were included and categorized, where appropriate. For physical activity (denoted $x_{1}$ ), for example, $\mathrm{x}_{1}=1$ if activity is frequent and 0 otherwise). The response variable $(y)$ was therefore modeled as a function of familial aggregation $(z)$, environmental risk factors $(x)$ and the interaction between them. As suggested by Liang and Pulver, the generalized estimation equation (GEE) method was used to estimate the regression coefficients for familial aggregation $(z)$ and environmental factors $(x)$ using SAS software (Version 8.2). The importance of familial aggregation was determined by assessing the magnitude of the related model coefficients $(z)$. Taking the exponential of $z$ gives the odds ratio for the risk of developing MET-S for family members among case probands compared with that among control probands. The higher the odds ratio (OR) is, the stronger the tendency to have familial aggregation. Adjusted odds ratios (aOR) and their respective 95\% CI's for environmental risk factors were also calculated in the same manner.

As our study unit is based on proband's family members, by categorizing familial aggregation in different ways, degree of relationship in the link with proband was ascertained and treated as an independent variable with eight types, mother, father, wife, husband, siblings, son, daughter, and second-degree relatives (grandparents, uncle, aunt, and cousins). As the majority of our study subjects were aged 30 years or older, few proband's sons and daughters were included. In addition, siblings of proband are also difficult to be ascertained because they may be separate and registered as different households after marriage in population registry. We only assessed the influence of mother, father, husband, and wife in comparison to seconddegree relatives. This has been also elaborated in Appendix A. In multiple regression analysis, we first incorporated significant factors identified in the univariate analysis together with age and gender of probands and then identified a parsimonious model by using the likelihood ratio test with a $5 \%$ significance level to exclude non-significant factors. To minimize the residual confounding, life style factors such as smoking, drinking, and betel quids chewing were classified as three categories: current, quit, and never. For the frequency of smoking, alcohol drinking, betel quids, and physical activity, tertile, quartile, or quintitle was first determined by using likelihood ratio test. As there was lacking of statistical significance across three types, tertile distributions were therefore selected to assess the magnitude of their associations. Note that we also tested whether the difference between five level and two level (usually/infrequent) for dietary factors. As there was lacking of statistical significance, we chose the dichotomous variable for analysis.

\section{Results}

\subsection{Prevalence rate of $M E T-S$}

Table 1 shows the age-specific prevalence rates of MET-S amongst the 43,158 attendants of the KCIS programme (using modified NCEP ATPIII criteria). The overall prevalence of MET-S using the standard NCEP ATPIII criteria was $10.6 \%$ (10.1\% in males and $10.9 \%$ in females). However, the application of modified NCEP ATPIII criteria yields $16.9 \%$. The prevalence rates of MET-S in families with more than two or unrelated participants are similar to the aggregated rate for all families (see Table 1). The prevalence of the individual conditions that make up MET-S was also similar between the full set of 32,522 households and the 9139 households with two or more participants (data not shown). This suggests that households with two or more participants are fairly representative of the full KCIS cohort with 43,158 subjects.

The comparisons of baseline variables between case and control group are presented in Table 2. The prevalence of MET-S in the case group was higher than that in the control group (16.2\% versus $9.9 \%)$. The case group had lower education level than the control group.

Table 1

Prevalence rate of MET-S in the KCIS study, by age

\begin{tabular}{|c|c|c|c|}
\hline $\begin{array}{l}\text { Age group } \\
\text { (years) }\end{array}$ & $\begin{array}{l}\text { Total attendants }{ }^{\mathrm{a}} \\
(N=43,158)\end{array}$ & $\begin{array}{l}\text { Family members } \geq 2^{\mathrm{a}} \\
(N=19,744)\end{array}$ & $\begin{array}{l}\text { Unrelated subjects }^{\mathrm{a}} \\
(N=23,414)\end{array}$ \\
\hline $20-29$ & $89 / 2523(3.5 \%)$ & $53 / 1574(3.4 \%)$ & $36 / 949(3.8 \%)$ \\
\hline $30-39$ & $563 / 8462(6.6 \%)$ & $263 / 3935(6.7 \%)$ & $300 / 4527(6.6 \%)$ \\
\hline $40-49$ & 1192/10,427 (11.4\%) & $521 / 4480(11.6 \%)$ & $671 / 5947(11.3 \%)$ \\
\hline $50-59$ & $1571 / 7749(20.3 \%)$ & $733 / 3664(20.0 \%)$ & $838 / 4085(20.5 \%)$ \\
\hline $60-69$ & $1978 / 7554(26.2 \%)$ & $883 / 3467(25.5 \%)$ & $1095 / 4087(26.8 \%)$ \\
\hline 70-79 & $1607 / 5260(30.5 \%)$ & $642 / 2151(29.9 \%)$ & $965 / 3109(31.0 \%)$ \\
\hline $80+$ & $308 / 1183(26.0 \%)$ & $130 / 473(27.5 \%)$ & $178 / 710(25.1 \%)$ \\
\hline Total & $7308 / 43,158(16.9 \%)$ & $3225 / 19,744(16.3 \%)$ & $4083 / 23,414(17.4 \%)$ \\
\hline Test for trend ( $p$-value) & $<0.0001$ & $<0.0001$ & $<0.0001$ \\
\hline
\end{tabular}

\footnotetext{
${ }^{\text {a }}$ MET-S case/total (\%).
} 
Table 2

Comparison of demographics, anthropometric, and biochemical factors between case and control group

\begin{tabular}{|c|c|c|c|}
\hline Variable & Class & Control & Case \\
\hline \multirow[t]{2}{*}{ MET-S } & Without MET-S & $2680(90.1)$ & $1438(83.8)$ \\
\hline & With MET-S & $294(9.9)$ & $278(16.2)$ \\
\hline \multirow[t]{2}{*}{ Gender } & Female & $1710(57.5)$ & 985 (57.4) \\
\hline & Male & $1264(42.5)$ & $731(42.6)$ \\
\hline \multirow{6}{*}{$\begin{array}{l}\text { Age group } \\
\quad \text { (years) }\end{array}$} & $20-29$ & $271(9.1)$ & $147(8.6)$ \\
\hline & $30-39$ & $590(19.8)$ & $295(17.2)$ \\
\hline & $40-49$ & $579(19.5)$ & $327(19.0)$ \\
\hline & $50-59$ & $522(17.6)$ & 290 (16.9) \\
\hline & $60-69$ & $545(18.3)$ & 338 (19.7) \\
\hline & $70+$ & $467(15.7)$ & 319 (18.6) \\
\hline \multirow[t]{4}{*}{ Education } & Low $(<6$ years $)$ & $1143(38.8)$ & $754(44.3)$ \\
\hline & Middle (6-12 years) & $1261(42.7)$ & $680(39.9)$ \\
\hline & High ( $\geq 12$ years $)$ & $547(18.5)$ & $268(15.8)$ \\
\hline & Unknown & 23 & 13 \\
\hline \multirow[t]{4}{*}{ Marriage } & Unmarried & $149(5.0)$ & $89(5.2)$ \\
\hline & Married & $2444(82.2)$ & $1411(82.2)$ \\
\hline & Divorced & $380(12.8)$ & $216(12.6)$ \\
\hline & Unknown & 1 & 0 \\
\hline \multirow{4}{*}{$\begin{array}{l}\text { Chewing } \\
\text { betel quids }\end{array}$} & Never & $2772(94.1)$ & 1595 (93.6) \\
\hline & Quit & $90(3.0)$ & $51(3.0)$ \\
\hline & Current & $85(2.9)$ & $58(3.4)$ \\
\hline & Unknown & 27 & 12 \\
\hline \multirow[t]{4}{*}{ Smoking } & Never & $2119(71.8)$ & $1207(70.7)$ \\
\hline & Quit & $224(7.6)$ & $103(6.0)$ \\
\hline & Current & $609(20.6)$ & $398(23.3)$ \\
\hline & Unknown & 22 & 8 \\
\hline \multirow[t]{4}{*}{ Drinking } & Never & $2268(77.3)$ & $1335(78.5)$ \\
\hline & Quit & $141(4.8)$ & $71(4.2)$ \\
\hline & Current & $525(17.9)$ & $295(17.3)$ \\
\hline & Unknown & 40 & 15 \\
\hline
\end{tabular}

\begin{tabular}{lcc} 
Variable & Control & \multicolumn{1}{c}{ Case } \\
\hline Waist circumferences & $78.3 \pm 10.8$ & $80.7 \pm 10.7$ \\
Body mass index (BMI) & $23.9 \pm 3.7$ & $24.5 \pm 3.8$ \\
Systolic blood pressure (SBP) & $124.6 \pm 20.8$ & $127.8 \pm 20.7$ \\
Diastolic blood pressure (DBP) & $76.9 \pm 11.7$ & $77.8 \pm 11.8$ \\
Fasting glucose & $92.6 \pm 24.9$ & $96.6 \pm 31.9$ \\
Triglyceride & $116.6 \pm 82.8$ & $127.7 \pm 92.4$ \\
High density lipoprotein (HDL) & $61.8 \pm 13.6$ & $59.8 \pm 13.4$ \\
\hline
\end{tabular}

The values in parentheses are given in percentage.

Regarding life style factors, there were higher proportions of current smokers and chewers in the case group than the control group. The comparisons of biochemical markers of MET-S are also specified in Table 2. The case group had higher mean values in BMI, waist circumferences, blood pressure, fasting glucose, triglyceride but lower in high density lipoprotein.

\subsection{Familial aggregation of MET-S with environmental factors being adjusted}

Table 3 shows odds ratio for having MET-S among family members for cases versus control probands was 1.70 -fold (95\% CI 1.41-2.04). This suggests that there is a strong tendency to familial aggregation in association with MET-S.

Repeating the analysis for parents and spouses of index cases and controls, the highest odds ratio was noted for mother $(\mathrm{OR}=3.17(1.99-5.06))$, followed by father $(\mathrm{OR}=2.23(1.10-4.54))$, husband $(\mathrm{OR}=1.61$ $(0.94-2.77))$ and wife $(\mathrm{OR}=1.37(0.98-1.91))$, when taking second-degree relatives as the baseline group.

Table 3 shows adjusted odds ratios for demographic variables, putative environmental factors and familial aggregation. The variables retained in the final model are listed in the final column of Table 3. After controlling for age, gender, and other significant factors of probands, the tendency to familial aggregation still remained statistically significant (adjusted odds ratio $=1.56(1.29-1.89)$ ).

By looking into the degree of relative relationship to index cases or control and taking second-degree relatives as the baseline group with making allowance for the environmental factors similar to those delineated in Table 3, the tendency of familial aggregation was the most remarkable for mother $(\mathrm{aOR}=1.55(0.98-2.45)$ ), which was nearly statistically significant. This tendency was attenuated for father $(\mathrm{aOR}=1.39(0.78-2.50))$, wife $\quad(\mathrm{aOR}=1.10 \quad(0.83-1.45)), \quad$ and husband $(\mathrm{aOR}=0.99(0.73-1.33))$.

\subsection{Familial aggregation associated with individual components of MET-S after adjustment for significant environmental factors}

Table 4 shows the corresponding results of individual components of MET-S. The tendency of familial aggregation was significantly manifested in each individual component with the order of adjusted odds ratios ranging from $1.28(1.01-1.48)$ for hypertriglyceridemia to $1.43(1.25-1.64)$ for obesity. Familial aggregation contributed from mother was significantly noted in central obesity $(\mathrm{aOR}=1.44(1.00-2.08))$ and nearly significant in hyperglycemia $(\mathrm{aOR}=1.57(0.97-$ 2.56)) after controlling for significant environmental factors (see footnote of Table 4). Familial aggregation contributed from spouse was also significantly noted in hyperglycemia $(\mathrm{aOR}=1.43(1.04-1.96))$ and high blood pressure $(\mathrm{aOR}=1.26(1.02-1.57))$ for husband, and in obesity $(\mathrm{aOR}=1.27(1.06-1.57))$ for wife after 
Table 3

Adjusted odds ratios for familial aggregation and environmental risk factors associated with MET-S

\begin{tabular}{|c|c|c|}
\hline Variable & $\mathrm{aOR}^{1}(95 \% \mathrm{CI})$ & $\mathrm{aOR}^{2}(95 \% \mathrm{CI})$ \\
\hline Familial aggregation & $1.70(1.41-2.04)^{\ddagger}$ & $1.56(1.29-1.89)^{\ddagger}$ \\
\hline Age of family members of proband & - & $1.04(1.03-1.05)^{\ddagger}$ \\
\hline Gender of family members of proband & - & $1.10(0.98-1.36)$ \\
\hline \multicolumn{3}{|l|}{ Socioeconomic status } \\
\hline Education (low/high) & $2.00(1.36-2.93)^{\dagger}$ & $2.06(1.36-3.13)^{\dagger}$ \\
\hline Education (middle/high) & $1.62(1.12-2.33)^{*}$ & $1.67(1.13-2.49){ }^{*}$ \\
\hline Marriage (divorced/unmarried) & $1.37(0.79-2.38)$ & \\
\hline Marriage (married/unmarried) & $1.12(0.72-1.73)$ & \\
\hline \multicolumn{3}{|l|}{ Reproductive factors } \\
\hline Postmenopausal (yes/no) & $1.40(0.93-2.13)$ & \\
\hline Age at menarche & $0.97(0.90-1.05)$ & \\
\hline Age at menopause & $1.06(1.00-1.12)^{*}$ & $1.08(1.01-1.14)^{*}$ \\
\hline Age at first full-term pregnancy & $0.94(0.90-0.98)^{\dagger}$ & $0.93(0.88-0.98)^{\dagger}$ \\
\hline Number of pregnancies & $1.04(0.98-1.11)$ & \\
\hline Parity & $1.05(0.97-1.14)$ & \\
\hline Number of abortions & $1.09(0.98-1.22)$ & \\
\hline \multicolumn{3}{|l|}{ Life style factors } \\
\hline \multicolumn{3}{|l|}{ Current/quit/never } \\
\hline Smoking (quit/never) & $0.70(0.47-1.04)$ & \\
\hline Smoking (current/never) & $1.05(0.81-1.35)$ & \\
\hline Drinking (quit/never) & $1.03(0.66-1.61)$ & \\
\hline Drinking (current/never) & $1.15(0.89-1.49)$ & \\
\hline Betel chewing (quit/never) & $2.01(1.22-3.33)^{\dagger}$ & $1.86(1.11-3.10)^{*}$ \\
\hline Betel chewing (current/never) & $2.27(1.37-3.76)^{\dagger}$ & $2.00(1.20-3.35)^{\dagger}$ \\
\hline \multicolumn{3}{|l|}{ Frequency/quantity } \\
\hline Smoking piece/day ( $<15$ pieces/never) & $0.84(0.62-1.14)$ & \\
\hline Smoking piece/day ( $\geq 15$ pieces/never) & $1.03(0.77-1.39)$ & \\
\hline Drinking/week ( $<2$ times/never) & $1.34(0.07-2.57)$ & \\
\hline Drinking/week ( $\geq 2$ times/never) & $1.48(0.90-2.43)$ & \\
\hline Betel pieces/day ( $<10$ pieces/never) & $2.07(1.18-3.63)^{*}$ & $1.99(1.13-3.53)^{*}$ \\
\hline Betel pieces/day ( $\geq 10$ pieces/never) & $2.09(1.16-3.79)^{*}$ & $1.76(0.96-3.23)$ \\
\hline Exercise frequency/week ( $<3 /$ none $)$ & $0.80(0.63-1.01)$ & $0.83(0.65-1.06)$ \\
\hline Exercise frequency/week ( $\geq 3 /$ none) & $0.66(0.53-0.83)^{\dagger}$ & $0.72(0.58-0.90)^{\dagger}$ \\
\hline \multicolumn{3}{|l|}{$\begin{array}{l}\text { Exercise intensity (times of } \\
\quad \text { per week }) \times(\text { minutes of per time })\end{array}$} \\
\hline Exercise intensity $(<100 \mathrm{~min} /$ none $)$ & $0.75(0.60-0.94)^{*}$ & $0.79(0.63-0.98)^{*}$ \\
\hline Exercise intensity ( $\geq 100 \mathrm{~min} /$ none $)$ & $0.69(0.55-0.86)^{\dagger}$ & $0.74(0.59-0.94)^{*}$ \\
\hline \multicolumn{3}{|l|}{ Dietary factors } \\
\hline Meat (usually/infrequent) & $1.08(0.89-1.30)$ & \\
\hline Fish (usually/infrequent) & $0.99(0.80-1.22)$ & \\
\hline Vegetable (usually/infrequent) & $0.68(0.56-0.81)^{\ddagger}$ & $0.76(0.62-0.92)^{\dagger}$ \\
\hline Fruit (usually/infrequent) & $0.88(0.71-1.09)$ & \\
\hline Bean (usually/infrequent) & $1.21(0.99-1.47)$ & \\
\hline Milk (usually/infrequent) & $0.99(0.82-1.20)$ & \\
\hline Coffee (usually/infrequent) & $1.50(1.23-1.83)^{\ddagger}$ & $1.32(1.07-1.64)^{*}$ \\
\hline
\end{tabular}

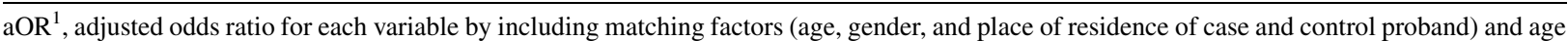
and gender of family members from each case or control proband in the model; $\mathrm{aOR}^{2}$, adjusted for familial aggregation and significant factors in each other.

* $0.01 \leq p<0.05$.

${ }^{\dagger} 0.0001 \leq p<0.01$.

$\ddagger p<0.0001$. 
Table 4

Adjusted odds ratios for familial aggregation associated with individual components of MET-S

\begin{tabular}{|c|c|c|c|c|c|}
\hline \multirow{2}{*}{$\begin{array}{l}\text { Components of } \\
\text { metabolic syndrome }\end{array}$} & \multirow{2}{*}{$\begin{array}{l}\text { Case probands/ } \\
\text { control probands }\end{array}$} & \multicolumn{4}{|c|}{ Adjusted odds ratio } \\
\hline & & Father & Mother & Husband & Wife \\
\hline Central obesity & $1.43(1.25-1.64)$ & $0.73(0.45-1.20)$ & $1.44(1.00-2.08)$ & $0.83(0.66-1.03)$ & $1.27(1.06-1.53)$ \\
\hline Triglyceride & $1.28(1.10-1.48)$ & $1.11(0.67-1.84)$ & $1.20(0.81-1.79)$ & $1.00(0.80-1.26)$ & $0.85(0.69-1.04)$ \\
\hline $\mathrm{HDL}$ & $1.33(1.08-1.63)$ & $1.21(0.57-2.57)$ & $1.18(0.67-2.08)$ & $0.65(0.46-0.94)$ & $1.10(0.85-1.43)$ \\
\hline High blood pressure & $1.25(1.09-1.43)$ & $1.37(0.82-2.28)$ & $1.00(0.69-1.46)$ & $1.26(1.02-1.57)$ & $0.79(0.66-0.94)$ \\
\hline Hyperglycemia & $1.28(1.06-1.56)$ & $1.31(0.71-2.44)$ & $1.57(0.97-2.56)$ & $1.43(1.04-1.96)$ & $1.24(0.91-1.69)$ \\
\hline
\end{tabular}

${ }^{a}$ Other independent variables controlled in the model included age, sex, education, and exercise intensity for central obesity; age, sex, coffee intake, betel chewing, education, and exercise intensity for triglyceride; age, sex, education, vegetable and coffee intake, alcohol drinking, and exercise intensity for HDL; age, sex, education, vegetable and coffee intake, alcohol drinking, and exercise intensity for high blood pressure; age, sex, coffee intake, and education for hyperglycemia.

controlling for other significant variables. It should be noted that some aOR estimates in Table 4 were less than 1 and significant. Recall that as the baseline group was second-degree relatives this should be taken with great caution. The significant results for adjusted odds ratio under 1 may suggest that second-degree relatives may have stronger familial aggregation tendency than spouse.

\section{Discussion}

The present family case-control proband study demonstrates that the occurrence of metabolic syndrome (MET-S) has a strong tendency to familial aggregation even after controlling for demographic features, significant environmental factors, and reproductive and menstrual factors. The cases (MET-S) had approximately 1.56 -fold risk of having family members with MET-S as the controls (non-MET-S). Higher risk for MET-S in parents than in spouses suggests genetic inheritance may play an important role in the development of MET-S although shared environmental factors cannot be completely ruled out.

It is very interesting to note that, in addition to established risk factors (i.e., exercise, dietary factors, and so on), betel quids chewing was found a significant risk factor for MET-S in our study. The association between betel chewing and MET-S has been reported in recent Taiwanese studies, including the higher risk for type 2 diabetes in chewers [25], transgenerational effect of paternal betel quids chewing on the risk for MET-S in offspring [26], positive associations between betel quids chewing and individual components of MET-S [27,28]. However, unlike the current study, these studies did not take familial aggregation of MET-S into account. After controlling for familial aggregation, chewing betel nuts less than 10 pieces/day was an independent predictor of
MET-S, but the association was not significant when the frequency of betel chewing was greater than 10 pieces/ day. The reason for being unable to show a statistically association for the frequency of betel chewing more than 10 pieces may be due to sparse number at the end of category. However, we further assessed whether the trend across three groups (none, $\leq 10$ pieces, and $>10$ pieces) was statistically significant by using trend test. The result was statistically significant for betel quids chewing $(p<0.001)$. This suggests a dose-response relationship as demonstrated in the previous study [27] even after adjustment for familial aggregation and other significant risk factors.

As far as familial aggregation is concerned, the current results support the earlier finding based on twin studies that the common factors contributing to the clustering of obesity is both genetic and environmental $[13,14]$. However, as the occurrence of familial aggregation reflects genetic influences as well as the impact of sharing the same environment, our results are plausible and consistent with earlier findings [29] that concluded that genetic and/or non-genetic familial influence make significant contribution to the development of MET-S. Although both studies cannot separate genetic factor from independent environmental factors each study has addressed familial aggregation in each own way. In Liese et al.'s study [29], the significant association between a parent history of MET-S components and the clustering of these metabolic disorders in the offspring generation has been demonstrated. In our study, as we included the proband's parents, spouses, and other second-degree relatives, we are able to assess the impact of each family member separately. The higher odds ratios found in parents compared with the spouse suggests that genetic inheritance plays a key role although we cannot rule out the possibility of lifestyle habits conferred by 
parents. When considering the individual components of MET-S separately, after controlling for individual environmental factors, maternal influence was shown to play a major role in the occurrence of central obesity and hyperglycemia but only a minor role as regards hyperlipidemia, hypertriglyceridemia, and hypertension. These findings are also consistent with earlier results from a population study of elderly twins [15], suggesting that genetic factors contributed more to BMI and insulin resistance than to triglycerides, HDL cholesterol, and systolic blood pressure. It is very interesting to note that that familial aggregation has an especially strong maternal component. In addition to the genetic influences as mentioned above, it may reflect the importance of childhood or intra-uterine exposures (Barker hypothesis) or a great influence of the mother.

In contrast to previous studies on familial aggregation and MET-S, which used a traditional family casecontrol design to recall family history regarding MET-S with a self-reported questionnaire, our study exploited the sampling design of the KCIS screening project to implement a case-control proband study. This design has several strengths. Firstly, instead of relying on recalled family histories, information was obtained directly by measuring and assessing all participants. This was rarely possible in previous studies. Thus there is no recall bias. Secondly, as the case and control probands were sampled from participants in a population-based screening programme, selection bias between the presence of MET-S among relatives and probands should be minimal or, at the very least, balanced out.

There are several limitations in the current study. First, our study can only elucidate familial aggregation and environmental factors simultaneously but cannot separate genetic factors from environmental factors because familial aggregation may be also due to unmeasured and shared environmental factors that were not collected in the current study. Aggregation in some individual components such as high blood pressure or hyperglycemia for spouse may account for the latter possibility. Table 4 also shows familial aggregation. This is particularly important for Chinese people because spouse, particularly wife, have much influence on dietary habits and life style that may or may not be measured in the current study. Second, even we collected a host of environmental factors but many important factors like the amount of fat in the diet that affects HDL-cholesterol concentrations or concentrations and types of alcohol are still not assessed. Third, since we only included few siblings, familial aggregation among siblings of probands cannot be assessed.
We concluded that cases of MET-S tend to aggregate within Taiwanese families even after controlling for established risk factors in association with MET-S. Maternal influence in familial aggregation of MET-S may suggest further genetic studies.

\section{Appendix A}

\section{A.1. Logistic regression model for case-control/ family sampling design}

Let $y_{i j}$ a binary outcome of the presence of metabolic Syndrome for $j$ th relatives of $i$ th proband, $X_{i j m}$ be the $m$ th component of $p$ environmental risk factor ( $X_{1}=$ coffee, $X_{2}=$ exercise, $\ldots, X_{p}=$ vegetable $)$ and $Z_{i}$ be the status of proband $(Z=1$, case proband; $Z=0$, control proband).

The logistic regression model for assessing familial aggregation and environmental risk factors terms of $Z$ and $X$, respectively, was expressed as follows:

$\log \left(\frac{P_{i j}}{1-P_{i j}}\right)=\alpha+\beta_{1} X_{1}+\cdots+\beta_{p} X_{p}+\delta Z_{i}$

Eq. (1) enables one to assess the independent contribution of familial aggregation from regression coefficient of $\delta$ after controlling for environmental risk factors $\left(X_{1}-X_{p}\right)$. While the relationship of family members in association with proband was considered the variable of $Z$ was recoded as four dummy variables to assess the influence of mother, father, wife, and husband on the risk for MET-S compared with seconddegree relatives.

\section{References}

[1] F.L. Brancati, P.K. Whelton, L.H. Kuller, et al., Diabetes mellitus, race, and socioeconomic status: a population-based study, Ann. Epidemiol. 6 (1996) 67-73.

[2] E.J. Brunner, M.G. Marmot, K. Nanchahal, et al., Social inequality in coronary risk: central obesity and the metabolic syndrome evidence from the Whitehall II study, Diabetologia 40 (1997) 1341-1349.

[3] M.A. Winkleby, H.C. Kraemer, D.K. Ahn, et al., Ethnic and socioeconomic differences in cardiovascular disease risk factors: finding for women from the third national health and nutrition examination survey 1988-1994, JAMA 280 (1998) 356-362.

[4] S.P. Wamala, J. Lynch, M. Horsten, et al., Education and the metabolic syndrome in women, Diab. Care 22 (1999) 19992003.

[5] T. Hidvegi, K. Hetyesi, L. Biro, et al., Education level and clustering of clinical characteristics of metabolic syndrome, Diab. Care 24 (2001) 2013-2014.

[6] D.A. Schoeller, K. Shay, R.F. Kushner, How much physical activity is needed to minimize weight gain in previously obese women? Am. J. Clin. Nutr. 66 (1997) 551-556. 
[7] K.L. Rennie, N. McCarthy, S. Yazdgerdi, M. Marmot, E. Brunner, Association of the metabolic syndrome with both vigorous and moderate physical activity, Int. J. Epidemiol. 32 (2003) 600606.

[8] J. Eriksson, S. Taimela, V.A. Koivisto, Exercise and the metabolic syndrome, Diabetologia 40 (1997) 125-135.

[9] R.M. Van Dam, E.B. Rimm, W.C. Willett, et al., Dietary patterns and risk for type 2 diabetes mellitus in U.A. men, Ann. Intern. Med. 136 (2002) 201-209.

[10] M.A. Pereira, D.R. Jacobs, L.V. Horn, et al., Dairy consumption, obesity, and insulin resistance syndrome in young adults: the CARDIA study, JAMA 287 (2002) 2081-2089.

[11] W. Bao, M.R. Srinivasan, W. Wattigney, G.S. Berenson, Persistence of multiple cardiovascular risk clustering related to syndrome $\mathrm{X}$ from childhood to young adulthood. The Bogalusa heart study, Arch. Intern. Med. 154 (1994) 1842-1847.

[12] P.T. Katzmarzyk, L. Perusse, R.M. Malina, J. Bergeron, J.P. Despres, C. Bouchard, Stability of indicators of the metabolic syndrome from childhood and adolescence to young adulthood: the Quebec family study, J. Clin. Epidemiol. 54 (2001) 190195.

[13] D. Carmelli, L.R. Cardon, R. Fabitz, Clustering of hypertension, diabetes, and obesity in adult male twins: same genes or same environments? Am. J. Hum. Genet. 55 (1994) 566-573.

[14] E.J. Mayer, B. Newman, M.A. Austin, D. Zhang, C.P. Quesenberry, K. Edwards, et al., Genetic and environmental influences on insulin levels and the insulin resistance syndrome: an analysis of women twins, Am. J. Epidemiol. 143 (1996) 323 332

[15] Y. Hong, H.L. Pedersen, K. Brismar, et al., Genetic and environmental architecture of the features of the insulin-resistance syndrome, Am. J. Hum. Genet. 60 (1997) 143-152.

[16] R. Koch, U. Julies, W. Jaross, H.E. Schroder, Estimation of the heritability of latent variables which are included in a structural model for metabolic syndrome, Hum. Heredity 52 (2001) 171176.

[17] R.J.F. Loos, P.T. Katzmarzyk, D.C. Rao, et al., Genome-wide linkage scan for the metabolic syndrome in the HERITAGE family study, J. Clin. Endocrinol. Metab. 88 (2003) 59355943.
[18] P. Poulsen, A. Vaag, K.O. Kyvik, et al., Genetic versus environmental aetiology of the metabolic syndrome among male and female twins, Diabetologia 44 (2001) 537-543.

[19] L.A. Sargeant, N.J. Wareham, K.T. Khaw, Family history of diabetes identifies a group at increased risk for the metabolic consequences of obesity and physical inactivity in EPIC-Norfolk: a population-based study, Int. J. Obes. 24 (2000) 13331339.

[20] T.H.H. Chen, Y.H. Chiu, D.L. Luh, et al., Community-based multiple screening model: design, implementation, and analysis of 42,387 participants, Cancer 100 (2004) 1734-1743.

[21] Executive Summary of the Third Report of the National Cholesterol Education Program (NCEP) Expert Panel on detection, evaluation, and treatment of high blood cholesterol in adults (Adult Treatment Panel III), JAMA 285 (2001) 2486-2497.

[22] World Health Organization, Obesity: Preventing and Managing the Global Epidemic, World Health Organization, Geneva, 2000 (WHO Technical Report Series, No. 894).

[23] World Health Organization, The Asia-Pacific Perspective: Redefining Obesity and its Treatment, WHO, Geneva, 2000.

[24] K.Y. Liang, A.E. Pulver, Analysis of case-control/family sampling design, Genet. Epidemiol. 13 (1996) 253-270.

[25] T.H. Tung, Y.H. Chiu, H.M. Wu, B.J. Boucher, T.H.H. Chen, A population-based study of the association between areca-nut chewing and type 2 diabetes mellitus in men (KCIS no. 2), Diabetologia 47 (2004) 1776-1781.

[26] T.H.H. Chen, Y.H. Chiu, B.J. Boucher, Transgenerational effects of betel quid chewing on the metabolic syndrome in the Keelung community-based integrated screening program, Am. J. Clin. Nutr. 83 (2006) 688-692.

[27] A.M.F. Yen, Y.H. Chiu, L.S. Chen, H.M. Wu, C.C. Huang, B.J. Boucher, et al., A population-based study on the association between betel quid chewing and the metabolic syndrome in men, Am. J. Clin. Nutr. 83 (2006) 1153-1160.

[28] J.Y. Guh, L.Y. Chuang, H.C. Chen, Betel-quid use is associated with the risk of the metabolic syndrome in adults, Am. J. Clin. Nutr. 83 (2006) 1313-1320.

[29] A.D. Liese, E.J. Mayer-Davis, H.A. Tyroler, et al., Familial components of the multiple metabolic syndrome: the ARIC study, Diabetologia 40 (1997) 963-970. 